\title{
Three-dimensional Volume Reconstruction Based on Trajectory Fusion from Confocal Laser Scanning Microscope Images
}

\author{
Sang-Chul Lee and Peter Bajcsy \\ National Center for Supercomputing Applications \\ University of Illinois at Urbana-Champaign, Urbana, IL \\ \{sclee,pbajcsy\}@ncsa.uiuc.edu
}

\begin{abstract}
In this paper, we address the problem of $3 D$ volume reconstruction from depth adjacent subvolumes (i.e., sets of image frames) acquired using a confocal laser scanning microscope (CLSM). Our goal is to align sub-volumes by estimating an optimal global image transformation which preserves morphological smoothness of medical structures (called features, e.g., blood vessels) inside of a reconstructed $3 D$ volume. We approached the problem by learning morphological characteristics of structures inside of each sub-volume, i.e. centroid trajectories of features. Next, adjacent sub-volumes are aligned by fusing the morphological characteristics of structures using extrapolation or model fitting. Finally, a global sub-volume to subvolume transformation is computed based on the entire set of fused structures. The trajectory-based $3 D$ volume reconstruction method described here is evaluated with a pair of consecutive physical sections using two evaluation metrics for morphological continuity.
\end{abstract}

\section{Introduction}

We address the problem of 3D volume reconstruction from depth adjacent sub-volumes (i.e., sets of image frames) acquired using confocal laser scanning microscopy (CLSM). CLSM is recognized as one of the major advances in microscopy due to its ability of non-destructive 3D imaging of a tissue with a relatively large thickness [1]. Especially, CLSM enables researchers to acquire images inside a cell at sub-cellular structures that was impossible using a conventional microscope. Nevertheless, there is a limit to the thickness of a physical section imaged by CLSM, and hence 3D volume reconstruction of CLSM data is still needed.
In general, 3D volume reconstruction is considered as a very challenging problem for structural and quantitative analyses, e.g., 3D surface area analysis and $3 \mathrm{D}$ visualization. The difference between 3D volume reconstructions from 2D histological cross sections acquired by CLSM and a regular bright-field microscope is in the presence of 3D structural information in CLSM sub-volumes (stacks of aligned image frames). Thus, 3D volume reconstruction from CLSM images can take advantage of the available 3D morphological information within each sub-volume as it is illustrated in this paper.

To construct a full 3D volume from multiple acquired sub-volumes, one would transform all 3D sub-volumes to a reference coordinate system. The underlying assumption in this process is based on the fact that CLSM imaging performs optical sectioning without changing geometric parameters during image acquisition. In other words, it is assumed that optical sections in a sub-volume are already perfectly aligned. On the contrary, physical sub-volumes could be rotated and translated due to specimen positioning, and possibly distorted by shear due to physical sectioning (tissue slicing and handling). It is usually very difficult or almost impossible to eliminate the slide preparation factors during acquisition process, and therefore an image transformation beyond rigid model is needed. Thus, our objective is to estimate the global affine transformation parameters by formulating the problem of $3 \mathrm{D}$ volume reconstruction from CLSM sub-volumes as an optimization problem that minimizes morphological discontinuity across physical boundaries of sub-volumes.

We approach the 3D volume reconstruction problem by learning morphological characteristics (such as centroid trajectories) of structures inside of each CLSM sub-volume first, and then aligning subvolumes by fusing the structures using their characteristics. The sub-volume alignment process is 


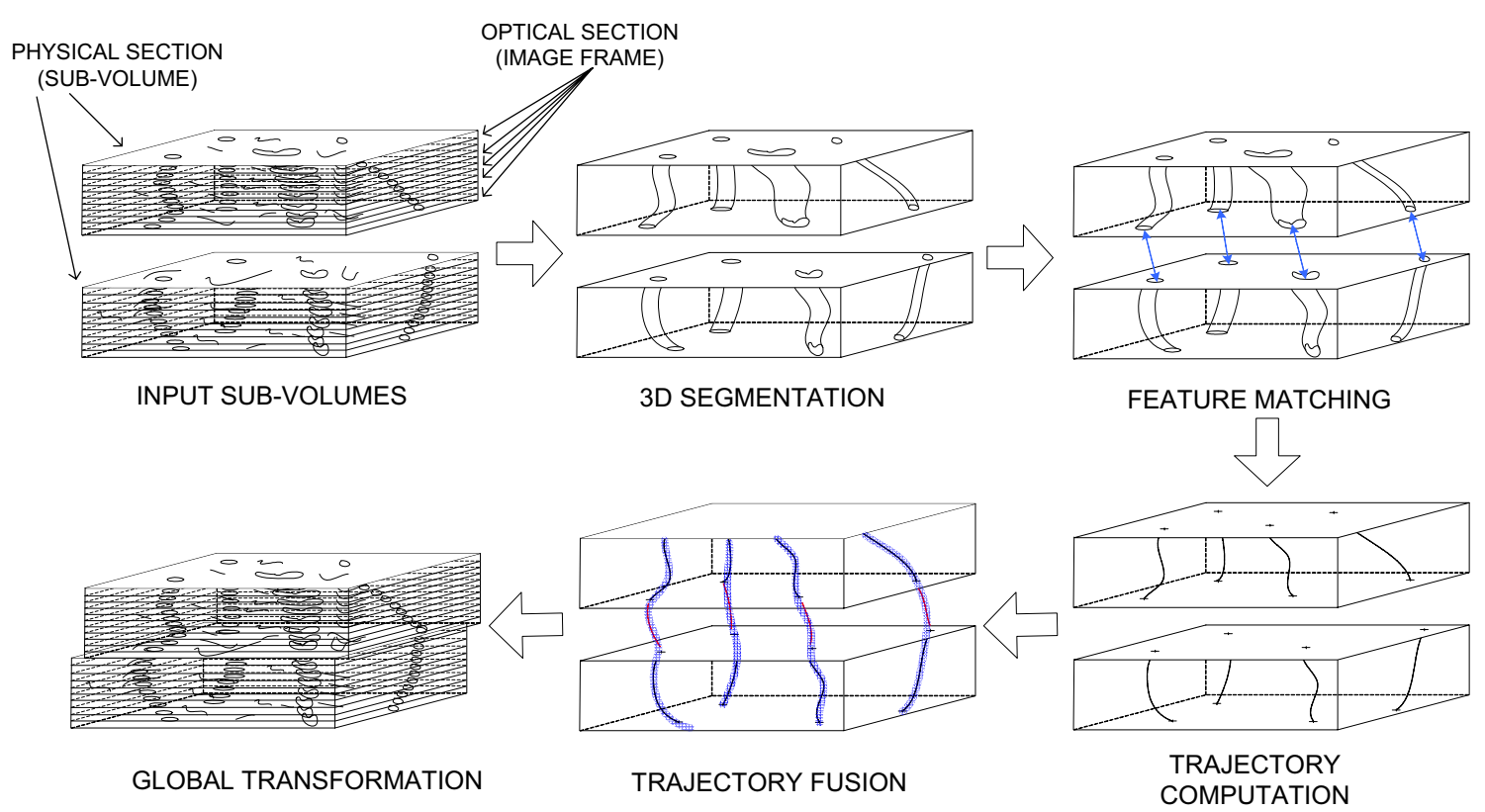

Figure 1: Overview of the trajectory-based alignment.

illustrated in Figure 1 as a sequence of the following steps; (1) segment out salient medical structures in each sub-volume, e.g., cylindrical structures (3D segmentation), (2) establish correspondences between structures from adjacent sub-volumes (feature matching), (3) estimate 3D trajectories from sets of points, e.g., from sets of $2 \mathrm{D}$ centroid points of $3 \mathrm{D}$ segments in each frame using regression-discontinuity analysis [2] (trajectory computation), (4) fuse two corresponding medical structures by combining their 3D trajectory models (trajectory fusion), and (5) compute a global sub-volume to sub-volume transformation based on the set of locally fused structures (global computation).

The previous methods developed for the problem of pair-wise sub-volume alignment can be classified as intensity based methods (e.g., normalized cross correlation or normalized mutual information) or morphological feature-based methods (e.g., shape matching, semi-automated method) [3]. Intensitybased methods are typically performed by selecting or generating a pair of representative images from adjacent sub-volumes [4], determining global transformation parameters by minimizing a similarity metric for all possible transformations, and applying the computed global transformation to the subvolume. Intuitively, using the intensity-based approach, one could select a pair of end frames that are near the boundary of adjacent physical sections to minimize morphological distortion of the structural changes. However, it is well known that due to spatial intensity heterogeneity of end frames in CLSM subvolumes, correlation techniques would result in very low similarity measures, which leads to undesirable sensitivity to noise and inaccurate alignments [5], [6]. To overcome the problems of intensity heterogeneity, alternative approaches for frame selection have been suggested that would select the highest contrast images [4] or generate a representative image based on sub-volume analysis, e.g., projection [7]. Although the intensity based methods are feasible in some cases, it should be noted that they are computationally very expensive due to a large search space of transformation parameters. If the search is limited then the alignment accuracy might be compromised and could result in discontinuities along z-axis (depth).

Given partial 3D information about structures presented in each CLSM sub-volume, we extracted centroid trajectories of volumetric segments for registration purposes. In the past, the use of similar trajectories fusion could be found in motion tracking problems from video sequences that were approached by the computer vision community [8]. In the case of video, trajectory fusion is performed by using all available spatio-temporal information [9]. Our work with 3D volumes differs in two aspects. First, trajectories are extracted from $2+\mathrm{z}$ dimensional data sets as opposed $2+t$ dimensional data sets. Second, the primary objective in motion tracking from video is to estimate each object motion from a trajectory, while the main objective in $3 \mathrm{D}$ volume reconstruction is to estimate a global registration transformation from all 
trajectories. Our trajectory-based approach to 3D volume reconstruction has not been explored for subvolume alignment problem in the past.

The paper is organized as followings: Section 2 describes the material preparation for test data and the process of $3 \mathrm{D}$ volume reconstruction using two different trajectory fusion methods defined by two evaluation methods. In Section 3, we present experimental results for different (a) trajectory fusion method, (b) physical gaps between adjacent subvolumes, and (c) polynomial model complexity.

\section{Methods}

In this paper, we developed a trajectory-based subvolume alignment method using two trajectory fusion objectives, such as minimum discontinuity across two adjacent sub-volumes and minimum residual of a polynomial fit to corresponding trajectory points from adjacent sub-volumes. The overall process of the developed techniques is illustrated in the previous section (Figure 1).

Material preparation and CLSM image acquisition were performed as following way: Formalin-fixed, paraffin-embedded uveal melanoma tissue samples were sectioned at $4 \mu \mathrm{m}$ thickness. All histological serial sections were examined with a Leica SP2 laser scanning confocal microscope (Leica, Heidelberg, Germany) using the $40 \times$ objective with $605 \sim 700 \mathrm{~nm}$ excitation wavelength range for the test specimens. Images were stored in tagged information file format (TIFF) with 512 by 512 pixel resolution. One 3D subvolume was formed from 13 image frames along axial coordinates (z-coordinate or depth) in a same lateral area. Therefore, a sub-volume consists of 512 by 512 by 13 voxels, which is equivalent to physical dimensions of $375 \times 375 \times 3.9 \mu \mathrm{m}$.

\subsection{Three-dimensional segmentation}

We developed a sphere-based region growing method that can segment out partially closed volumetric segments by extending the $2 \mathrm{D}$ disc-based segmentation method [4] to 3D sphere-based approach. In the absence of artificially inserted fiduciary markers, the volumetric segments in our data correspond to vascular structures used for registration. The fact that vascular structures are observed as partially closed volumetric segments in CLSM images is caused by photo bleaching or loss (possibly lack) of a fluorescent dye. The developed region growing method can recover vascular regions in those cases when an edge of a color homogeneous closed region is

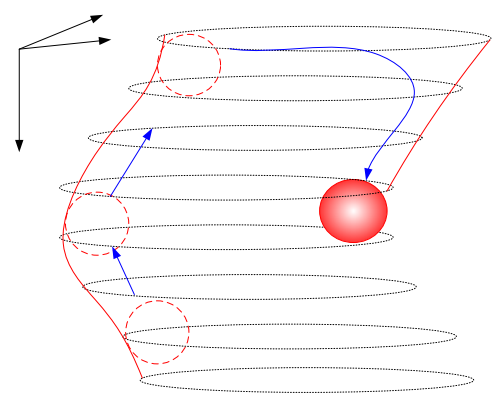

Figure 2: 3D segmentation of a volumetric region using a virtual sphere.

discontinued, an edge is partially destroyed during noise thresholding (especially if a high threshold value has to be applied to remove background noise), or an edge is missing since it lies partially outside of the imaged area. Figure 2 shows an illustration of 3D segmentation by growing interior region of a vascular region using a virtual sphere (ball).

\subsection{Selection of $3 D$ segments}

The three-dimensional segmentation method presented in Section 2.1 generates a set of $3 \mathrm{D}$ segments from a CLSM sub-volume. These segments may significantly deviate from a cylindrical model of a blood vessel. We removed some of the deviating segments by limiting the number of voxels in a volumetric structure. Although 3D segments with significant morphological changes or bifurcations may be interesting for clinical inspections, they are not necessarily useful in terms of registration purpose because of their complexity and trajectory uncertainty.

\subsection{Finding corresponding 3D segments}

The correspondence problem for 3D segments from adjacent sub-volumes is often easier to solve using shape characteristics of $2 \mathrm{D}$ cross sections of $3 \mathrm{D}$ segments rather than the shape characteristics of $3 \mathrm{D}$ segments. Our assumption is that the shape characteristics of 2D cross sections are sufficient to establish correspondences of 3D segments as proposed in [4] by solving a variation of the Procrustes problem [10]. We used the correspondence technique in [4] that is based on matching with the Euclidian distance based method followed by matching with the vector distance based method. Figure 3 shows an example of result for feature correspondence problem. 

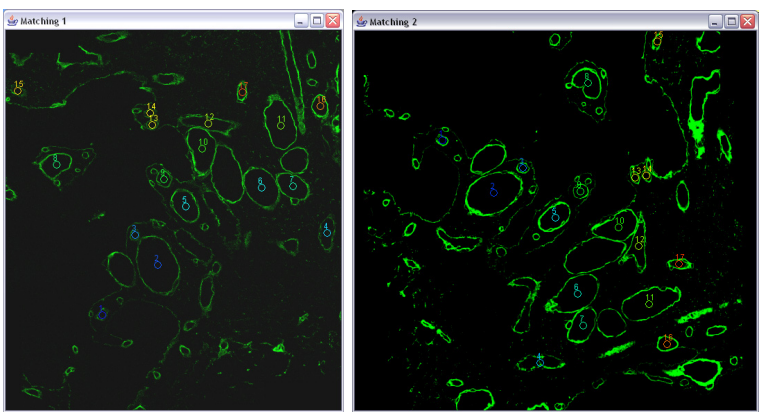

Figure 3: The feature correspondence for a pair of images to be aligned: The centroid locations of the segments are labeled for corresponding feature segments.

\subsection{Fusing corresponding sets of trajectory points}

The goal of this computation is (a) to calculate trajectory points of a 3D segment by estimating $2 \mathrm{D}$ centroids in each 2D frame of one sub-volume, and (b) to determine pairs of alignment control points from adjacent sub-volumes by fusing corresponding sets of trajectory points.

We define a set of 3D trajectory points derived for the $i$-th 3D segment in the $k$-th sub-volume as:

$$
t_{i}^{k}=\left\{\left(x_{1}, y_{1}, z_{1}\right),\left(x_{2}, y_{2}, z_{2}\right), \ldots,\left(x_{m}, y_{m}, z_{m}\right)\right\}
$$

where $m$ is the number of frames (depth) in the subvolume. The lateral coordinate $(x, y)$ is a centroid location in a frame, and the axial coordinate $z$ represents the depth in a 3D segment.

Next, we determine points of a pair of aligned trajectories. This task is achieved by performing trajectory fusion under a set of optimization objectives. The goal of trajectory fusion is to determine the pairs of control points to estimate the most accurate global transformation $\alpha: \mathbb{R}^{2} \rightarrow \mathbb{R}^{2}$ applied to lateral planes (frames) of sub-volumes. In our work, we chose to estimate affine transformation parameters.

First, given a set of matching pairs of depthadjacent trajectories $t_{i}^{k}$ and $t_{i}^{k+1}$, we compute $t_{i}^{* k+1}$ (see equation (2)) by one of the trajectory fusion methods described in the following sections (section 2.4.1 and section 2.4.2).

$$
\begin{aligned}
t_{i}^{* k+1}=\{ & \left(x_{1}+u_{i}, y_{1}+v_{i}, z_{1}\right), \\
& \left(x_{2}+u_{i}, y_{2}+v_{i}, z_{2}\right), \ldots, \\
& \left.\left(x_{m}+u_{i}, y_{m}+v_{i}, z_{m}\right)\right\}
\end{aligned}
$$

Then, $t_{i}^{* k+1}$ is considered as a set of modified (translated in lateral plane) trajectories that best fuse (connect) the corresponding features in $t_{i}^{k}$. Next, we compute the global affine transformation $\alpha$ by using all features based on the least-squares fit method defined as follows:

$$
\alpha(\cdot)=\underset{\alpha(\cdot)}{\arg \min }\left(\sum_{i=1}^{\mathrm{n}}\left[t_{i}^{* k+1}-\alpha\left(t_{i}^{k+1}\right)\right]^{2}\right)
$$

where $n$ is the number of matching 3D segments. Finally, we transform a set of images (frames) in the sub-volume $k+1$ by the estimated transformation $\alpha$ to create the new aligned sub-volume.

We developed two methods for trajectory fusion denoted as fusion by extrapolation and fusion by polynomial model fitting as described next.

2.4.1. Fusion by extrapolation. The trajectory fusion by extrapolation is driven by maximizing continuity of matching trajectories at the sub-volume border. Assuming that there exist some gaps between adjacent sub-volumes, we extrapolate a pair of points $\left(x_{m+1}^{k}, y_{m+1}^{k}, z_{m+1}^{k}\right) \in t_{i}^{k} \quad$ and $\quad\left(x_{0}^{k+1}, y_{0}^{k+1}, z_{0}^{k+1}\right) \in t_{i}^{k+1}$, where $z_{m}^{k}<z_{m+1}^{k}=z_{0}^{k+1}<z_{1}^{k+1}$. Then, we compute the translational offset $\left(u_{i}, v_{i}\right)$ of feature $i$ as follows:

$$
\left(u_{i}, v_{i}\right)=\left(x_{0}^{k+1}-x_{m+1}^{k}, y_{0}^{k+1}-y_{m+1}^{k}\right)
$$

To generate the pair of extrapolated points $\left(x_{m+1}^{k}, y_{m+1}^{k}, z_{m+1}^{k}\right)$ and $\left(x_{0}^{k+1}, y_{0}^{k+1}, z_{0}^{k+1}\right)$, we used two different methods, such as an end-point duplication and polynomial-based extrapolation with three different polynomial degrees. First, the end-point duplication is performed by replicating $(x, y)$ coordinate of the adjacent end trajectory points as illustrated below:

$$
\begin{aligned}
& \left(x_{m+1}^{k}, y_{m+1}^{k}, z_{m+1}^{k}\right)=\left(x_{m}^{k}, y_{m}^{k}, z_{m}^{k}+\Delta_{\text {gap }}\right) \\
& \left(x_{0}^{k+1}, y_{0}^{k+1}, z_{0}^{k+1}\right)=\left(x_{1}^{k+1}, y_{1}^{k+1}, z_{1}^{k+1}-\Delta_{\text {gap }}\right)
\end{aligned}
$$

An example of trajectory fusion by end-point duplication is illustrated in Figure 4 (a).

Second, the polynomial-based extrapolation is achieved by estimating the degree $\gamma$ of polynomial functions $f_{i}^{k(\gamma)}$ and $f_{i}^{k+1(\gamma)}$ based on $t_{i}^{k}$ and $t_{i}^{k+1}$. To compute a 3D polynomial curve, we estimate a function $f_{i}^{k(\gamma)}$ according to the equations below:

$$
\begin{aligned}
& f_{i x}^{k(\gamma)}(z)=a_{0}+a_{1} z+\ldots+a_{\gamma} z^{\gamma} \\
& f_{i y}^{k(\gamma)}(z)=b_{0}+b_{1} z+\ldots+b_{\gamma} z^{\gamma}
\end{aligned}
$$




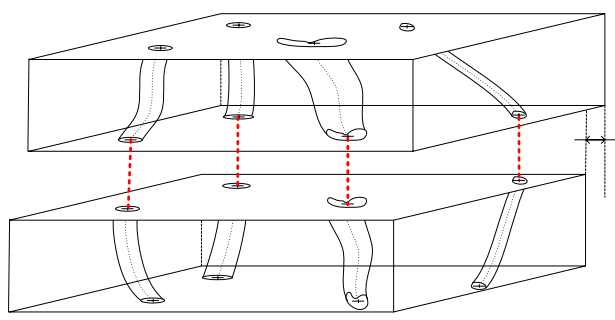

(a)

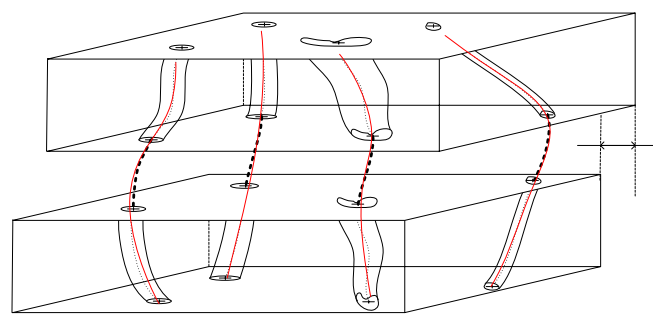

(b)

Figure 4: Trajectory fusion by different methods: (a) trajectory fusion by connecting end-points, and (b) trajectory fusion by residual minimization. $T_{1}$ and $T_{2}$ indicate the estimated transformation by extrapolation (end-point connection) and residual minimization method respectively.

where

$$
\begin{aligned}
& {\left[\begin{array}{cccc}
m & \sum_{j=1}^{m} z_{j} & \cdots & \sum_{j=1}^{m} z_{j}^{\gamma} \\
\sum_{j=1}^{m} z_{j} & \sum_{j=1}^{m} z_{j}^{2} & \cdots & \sum_{j=1}^{m} z_{j}^{\gamma+1}{ }_{j} \\
\vdots & \vdots & \ddots & \vdots \\
\sum_{j=1}^{m} z_{j}^{\gamma} & \sum_{j=1}^{m} z_{j}^{\gamma+1} & \cdots & \sum_{j=1}^{m} z_{j}^{2 \gamma}
\end{array}\right]\left[\begin{array}{cc}
a_{0} b_{0} \\
a_{1} b_{1} \\
\vdots & \vdots \\
a_{\gamma} b_{\gamma}
\end{array}\right]} \\
& =\left[\begin{array}{cc}
\sum_{j=1}^{m} x_{j} & \sum_{j=1}^{m} y_{j} \\
\sum_{j=1}^{m} z_{j} x_{j} & \sum_{j=1}^{m} z_{j} y_{j} \\
\vdots & \vdots \\
\sum_{j=1}^{m} z_{j}^{\gamma} x_{j} & \sum_{j=1}^{m} z_{j}^{\gamma} y_{j}
\end{array}\right] .
\end{aligned}
$$

Next, a pair of extrapolated points is generated as follows:

$$
\begin{gathered}
\left(x_{m+1}^{k}, y_{m+1}^{k}, z_{m+1}^{k}\right)=\left(f_{i x}^{k(\gamma)}\left(z_{m}^{k}+\Delta_{\text {gap }}\right),\right. \\
\left.f_{i y}^{k(\gamma)}\left(z_{m}^{k}+\Delta_{\text {gap }}\right), z_{m}^{k}+\Delta_{\text {gap }}\right) ; \\
\left(x_{0}^{k+1}, y_{0}^{k+1}, z_{0}^{k+1}\right)=\left(f_{i x}^{k+1(\gamma)}\left(z_{1}^{k+1}-\Delta_{\text {gap }}\right),\right. \\
\left.f_{i y}^{k+1(\gamma)}\left(z_{1}^{k+1}-\Delta_{\text {gap }}\right), z_{1}^{k+1}-\Delta_{\text {gap }}\right)
\end{gathered}
$$

Finally the translational offset $\left(u_{i}, v_{i}\right)$ of feature $i$ is computed as shown in equation (4).

2.4.2. Fusion by model fitting. The trajectory fusion by model fitting is driven by maximizing smoothness of matching trajectories within the two adjacent subvolumes. We assume relatively short trajectories of a blood vessel within each sub-volume follow a polynomial curve with the degree of less than three (cubic). Although the exact trajectory model and degree are not known, our results demonstrate a good cubic fit experimentally.
First, we define $r^{(\gamma)}\left(t_{i}^{k}\right)$ as a residual after fitting a polynomial function of $\gamma$-degree $f_{i}^{k(\gamma)}$ to a trajectory $t_{i}^{k}$ (see below):

$$
r^{(\gamma)}\left(t_{i}^{k}\right)=\left(r_{x}^{(\gamma)}\left(t_{i}^{k}\right)+r_{y}^{(\gamma)}\left(t_{i}^{k}\right)\right) / 2
$$

where $\quad r_{x}^{(\gamma)}\left(t_{i}^{k}\right)=\sqrt{\frac{1}{m} \sum_{j=0}^{m}\left(x_{i j}-f_{i x}^{k(\gamma)}\left(z_{i}\right)\right)^{2}}$ and

$$
r_{y}^{(\gamma)}\left(t_{i}^{k}\right)=\sqrt{\frac{1}{m} \sum_{j=0}^{m}\left(y_{i j}-f_{i y}^{k(\gamma)}\left(z_{i}\right)\right)^{2}} .
$$

To compute the residual of a fused trajectory from a pair of trajectories, we introduce a binary operator $\oplus_{\Delta_{k}}$ which merges a pair of trajectories between the sub-volumes $k$ and $k+1$, and with a physical gap $\Delta_{k}$ corresponding to missing frames. The parameter $\Delta_{k}$ is assumed to be constant for all sub-volumes in one set of experimental sub-volumes and $\Delta_{k}$ is typically less than the thickness spanned by three optical frames. The residual of a fused trajectory is then evaluated as $r^{(\gamma)}\left(t_{i}^{k} \oplus_{\Delta_{k}} t_{i}^{k+1}\right)$.

For each pair of matching trajectories $i$, we search for the translational offset $\left(u_{i}, v_{i}\right)$ of $t_{i}^{* k+1}$ by minimizing the residual $r^{(\gamma)}\left(t_{i}^{k} \oplus_{\Delta_{k}} t_{i}^{k+1}\right)$ :

$$
\left(u_{i}, v_{i}\right)=\underset{\left(u_{i}, v_{i}\right) \in \text { neighborhood }}{\arg \min }\left(r^{(\gamma)}\left(t_{i}^{k} \oplus_{\Delta_{k}} t_{i}^{* k+1}\right)\right)
$$

An example of trajectory fusion by residual minimization is shown in Figure 4 (b).

\subsection{Estimation of alignment transformation}


For the selected affine transformation model $\alpha(\cdot)$, the affine parameters can be estimated by choosing at least three pairs of corresponding points and computing six affine transformation parameters shown in equation below.

$$
\left[\begin{array}{l}
x^{\prime} \\
y^{\prime}
\end{array}\right]=\left[\begin{array}{ll}
\beta_{00} & \beta_{01} \\
\beta_{10} & \beta_{11}
\end{array}\right]\left[\begin{array}{l}
x \\
y
\end{array}\right]+\left[\begin{array}{l}
\beta_{02} \\
\beta_{12}
\end{array}\right]
$$

The values $\left(x^{\prime}, y^{\prime}\right)=\alpha(x, y)$ are the transformed coordinates of the original image coordinates $(x, y)$ by affine transformation $\alpha(\cdot)$. The parameter estimation can be performed either by a feature selection method, e.g., using a compactness measure [11], or by a least-squares fit method for estimating affine transformation parameters derived from corresponding trajectory points. In our work, we used the latter approach.

\subsection{Evaluation metrics for alignment accuracy}

We define two evaluation metrics for trajectory alignment accuracy, such as global residual and discontinuity. The evaluations are performed after applying the final global transformation.

Global residual metric: The global residual can be calculated as follows:

$$
R_{k}^{(\gamma)}=\frac{1}{\mathrm{n}} \sum_{i=1}^{n} r_{\mathrm{i}}^{(\gamma)}\left(t_{i}^{k} \oplus_{\Delta_{k}} \alpha\left(t_{i}^{k+1}\right)\right)
$$

where $r_{\mathrm{i}}^{(\gamma)}\left(t_{i}^{k} \oplus_{\Delta_{k}} \alpha\left(t_{i}^{k+1}\right)\right)$ is a residual of the $i$-th trajectory in sub-volumes $k$ and $k+1$, and $n$ is the number of matching trajectories (features). We assume that the sub-volume feature correspondence is known, for example, $t_{i}^{k}$ and $t_{i}^{k+1}$ are assumed to be a pair of matching volume features.

Discontinuity metric: We define a trajectory discontinuity $d\left(t_{i}^{k}, t_{i}^{k+1}\right)$ as a 2D $L^{2}$ distance (in lateral plane) of the pair of points that connect the adjacent trajectories.

$$
d\left(t_{i}^{k}, t_{i}^{k+1}\right)=\left\|e_{\text {last }}\left(t_{i}^{k}\right)-e_{\text {first }}\left(t_{i}^{k+1}\right)\right\|
$$

where $e_{\text {last }}\left(t_{i}^{k}\right)$ and $e_{\text {first }}\left(t_{i}^{k+1}\right)$ are the adjacent (the last of $t_{i}^{k}$ and the first of $t_{i}^{k+1}$ ) 2D lateral coordinates. To incorporate the sub-volume gap $\Delta_{k}$ for end point evaluations, the axial $z$-coordinates need to be

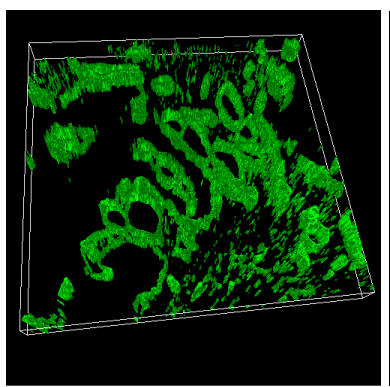

(a)

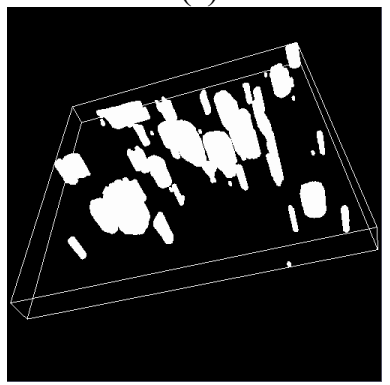

(c)

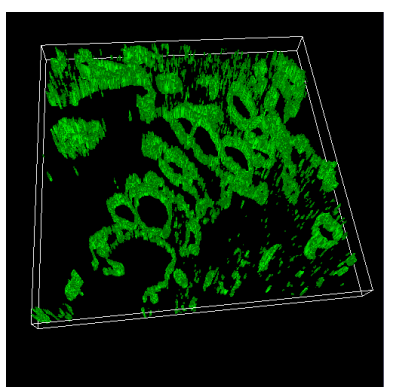

(b)

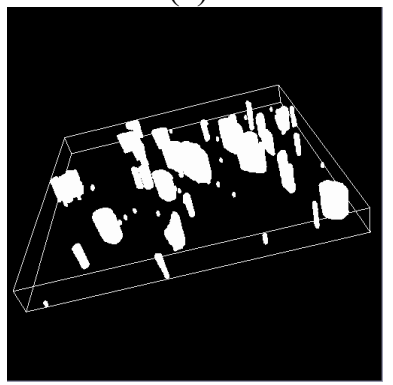

(d)
Figure 5: A pair of CLSM sub-volumes: (a) sub-volume 1 (upper physical section), (b) sub-volume 2 (lower physical section), (c) 3D segments of (a), and (d) 3D segments of (b).

adjusted by $+\left(\Delta_{k}+1\right) \times 0.5$ and $-\left(\Delta_{k}+1\right) \times 0.5$ for $t^{k}$ and $t^{k+1}$ respectively.

Finally, the global discontinuity $D_{k}$ of subvolumes $k$ and $k+1$ is defined as follows:

$$
D_{k}=\frac{1}{\mathrm{n}} \sum_{i=1}^{n} d\left(t_{i}^{k}, t_{i}^{k+1}\right)
$$

\section{Experimental Results}

We evaluated the global residual and discontinuity while considering three experimental variables for trajectory-based 3D volume reconstruction from CLSM: (1) the polynomial degree $\gamma$ of centroid trajectories along axial direction (z-axis of subvolumes), (2) the thickness of gaps between adjacent sub-volumes $\Delta_{k}$, (3) the trajectory fusion method (extrapolation and residual minimization).

Trajectory computation: Figure 5 shows a pair of input sub-volumes and segmented sub-volumes. After extracting volumetric segments, feature correspondences are established according to [4]. Next, trajectories $t_{i}^{\text {sub-volume } 1}$ and $t_{i}^{\text {sub-volume2 }}$ are established by computing centroids from the $i$-th 


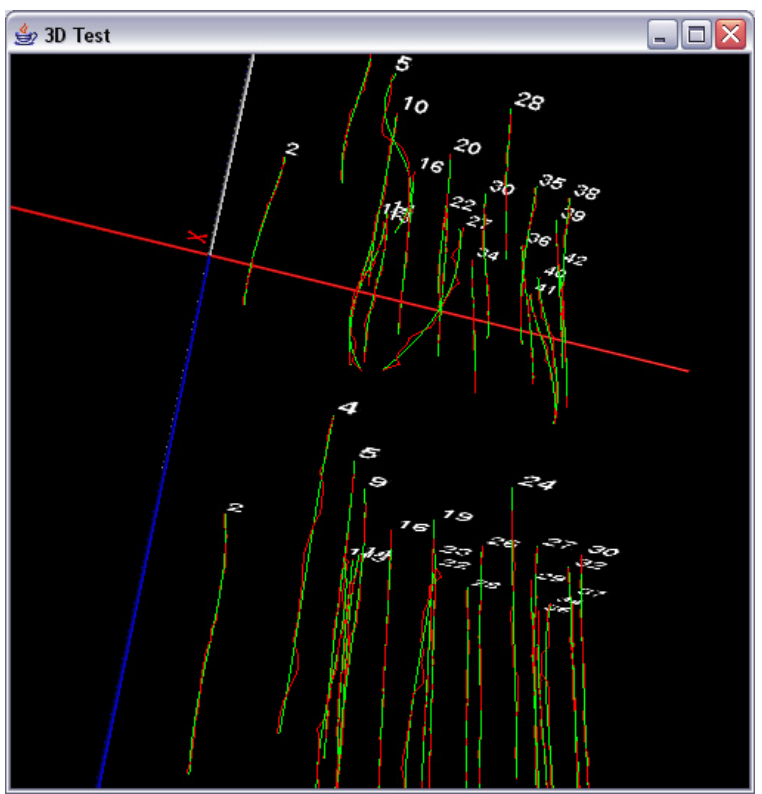

Figure 6: Sets of (exaggerated) trajectories from adjacent sub-volumes in Figure 5 . Numbers indicate the labels of matching trajectories. Red line segments represent centroids points, and green curves represents trajectories by polynomial fitting (cubic).

matching sub-volume features in all 2D optical sections (frames). An example pair of 3D trajectories is shown in Figure 6.

Trajectory fusion: Figure 7 shows the result of discontinuities from trajectory fusion using extrapolation method and residual minimization method with linear, quadratic, and cubic models and with respect to different sub-volume gaps. Based on the result, we concluded the following; (1) the extrapolation method achieves lower (better) discontinuity than the residual minimization method regardless of the polynomial degree (model complexity); (2) discontinuity is not necessarily related to the size of the physical gaps; and (3) discontinuity is relatively insensitive to the polynomial degree for each fusion method (e.g., curves in the graph using the same method remain close to each other).

Figure 8 shows the result of trajectory residuals after fusing depth-adjacent trajectories using extrapolation method and residual minimization method. From this result, we concluded that; (1) the residual minimization method always achieves lower (better) residual than the extrapolation method for the same polynomial degree; (2) all residuals decrease

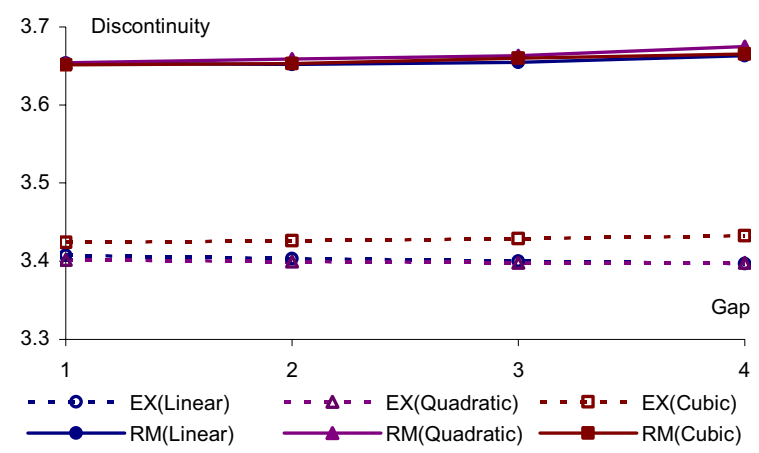

Figure 7: Trajectory discontinuity as function of physical gaps between adjacent subvolumes ${ }^{1}$.

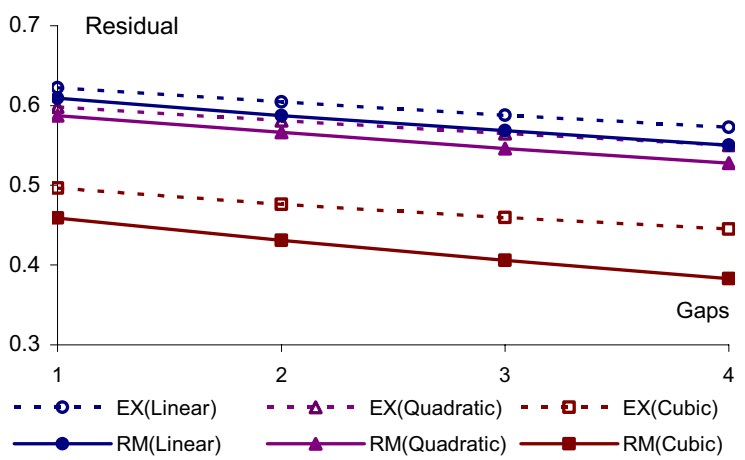

Figure 8: Trajectory residuals as a function of physical gaps between adjacent subvolumes ${ }^{1}$.

with the size of physical gaps of the adjacent subvolumes regardless of the fusion method and the polynomial degree; and (3) the resulting residual is more sensitive to the choice of the polynomial degree (model complexity) than to the choice of the fusion method.

Figure 9 shows some examples of the final threedimensional volumes without trajectory fusion (Figure 9 (b)), with trajectory fusion based on extrapolation method (Figure 9 (c)), and with trajectory fusion based on residual minimization method (Figure 9 (d)) respectively for the sub-area in Figure 9 (a). From sub-area views, one could visually verify that trajectory fusion by either method improves the discontinuity of registered volumetric features. Figure 9 (c) and (d) show different registration results based on different trajectory fusion approaches.

1 EX() and $\mathrm{RM}()$ refer to extrapolation method and residual minimization method respectively. Note that extrapolation method is 


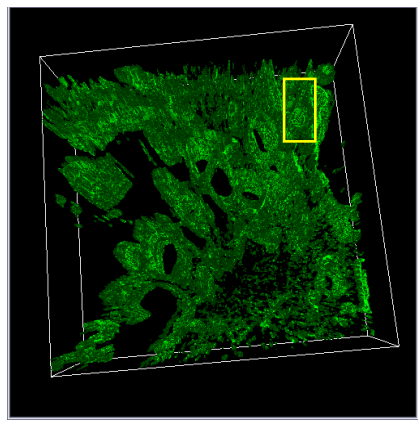

(a)

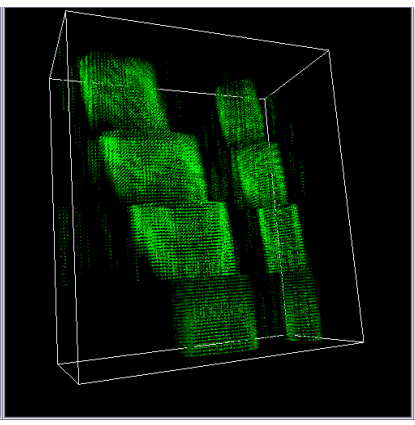

(b)

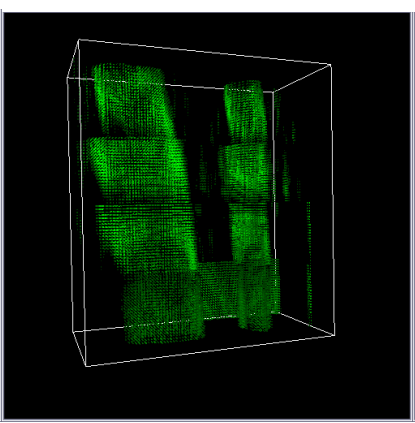

(c)

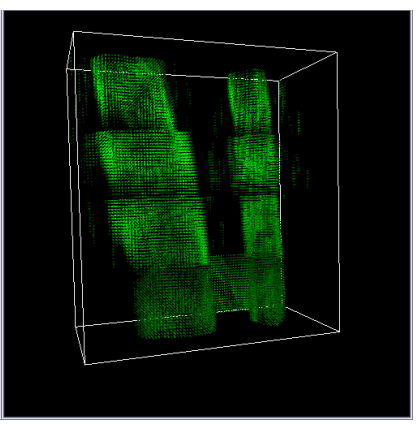

(d)

Figure 9: (a) Final reconstructed 3D volume: (b) by no trajectory fusion for gap $=1$, (c) by trajectory fusion based on extrapolation method, and (d) by trajectory fusion based on residual minimization method. Note that (b), (c), and (d) show side-views of the marked region (yellow box) in (a).

\section{Conclusion}

We addressed the problem of $3 \mathrm{D}$ volume reconstruction from depth adjacent CLSM subvolumes by estimating an optimal global image transformation which preserves morphological smoothness of medical structures inside of the reconstructed 3D volume. To preserve morphological smoothness of the reconstructed 3D medical structures, we defined two metrics for morphological continuity labeled as end point discontinuity and polynomial curve fitting residual, and then minimized the metrics across adjacent sub-volumes and all salient structures.

Finally, we discuss how an end-user could select the most appropriate fusion method and polynomial degree (model complexity). In terms of the fusion method selection, the choice should be driven by weighting priorities of continuity of matching trajectories at the sub-volume border or smoothness of matched trajectories within the adjacent sub-volumes. These decisions can be guided by designing a cost function for a hybrid fusion method. In terms of the polynomial degree selection, the polynomial degree (model complexity) is important if the trajectory model is known a priori and the residual is the main concern of an end-user. It is recommended to eliminate over-fitting by performing thorough studies of the medical structures of interest.

\section{References}

[1] J. Stevens, "Introduction to Confocal ThreeDimensional Volume Investigation", in three-dimensional

plotted as dotted lines, and residual minimization method is plotted by solid lines. confocal microscopy: volume investigation of biological systems, Academic press, pp. 3-27, 1994.

[2] W. M. Trochim, "The Research Methods Knowledge Base, 2e ", Cornell University, 2001.

[3] P. Bajcsy, S.-C. Lee, and D. Clutter, "Supporting Registration Decision during 3D Medical Volume Reconstruction", SPIE International Symposium in Medical Imaging, 6144-119, San Diego, 2006.

[4] P. Bajcsy, S.-C. Lee, A. Lin, and R. Folberg, "3D Volume Reconstruction of Extracellular Matrix Proteins in Uveal Melanoma from Fluorescent Confocal Laser Scanning Microscope Images", in Journal of Microscopy, Blackwell Synergy, vol. 221(1), pp. 30-45, 2006.

[5] D. Benson, J. Bryan, A. Plant, A. Gotto, and L. Smith, "Digital Imaging fluorescence microscopy: Spatial heterogeneity of photobleaching rate constants in individual cells", Journal of Cell Biology, vol. 100, pp. 1309-1323, 1985.

[6] M. Jungke, W. Seelen, G. Bielke, S. Meindl, et. al. "A system for the diagnostic use of tissue characterizing parameters in NMR-tomography", Proc. of Information Processing in Medical Imaging (IPMI'87), vol. 39, pp. 471481, 1987.

[7] H. Chan and A. Chung, "Efficient 3D-3D Vascular Registration Based on Multiple Orthogonal 2D Projections", Second International Workshop on Biomedical Image Registration (WBIR'03), pp.301 - 310, 2003.

[8] J. Kang, I. Cohen, and G. Medioni "Continuous multiview tracking using tensor voting", IEEE Workshop on Motion and Video Computing, Florida. 2002.

[9] Y. Caspi and M. Irani. "A Step towards Sequence-toSequence Alignment," IEEE Conference on Computer Vision and Pattern Recognition (CVPR'00), vol. 2, pp. 682689,2000

[10] L. Dorst, "First order error propagation of the procrustes method for 3D attitude estimation", IEEE Transactions on Pattern Analysis and Machine Intelligence. vol. 27(2), pp. 221- 229, 2005.

[11] S.-C. Lee and P. Bajcsy, "Feature based Registration of Flourescent LSCM Imagery”, in Proc. of SPIE International Symposium in Medical Imaging, vol. 5747, pp. 170-181, 2005. 